Document downloaded from:

http://hdl.handle.net/10251/147310

This paper must be cited as:

Badia, J.; Teruel Juanes, R.; Acebo, C.; Gil-Castell, O.; Serra, A.; Ribes-Greus, A. (2019). Dielectric spectroscopy of novel thiol-ene/epoxy thermosets obtained from allyl-modified hyperbranched poly(ethyleneimine) and diglycidylether of bisphenol A. European Polymer Journal. 113:98-106. https://doi.org/10.1016/j.eurpolymj.2019.01.001

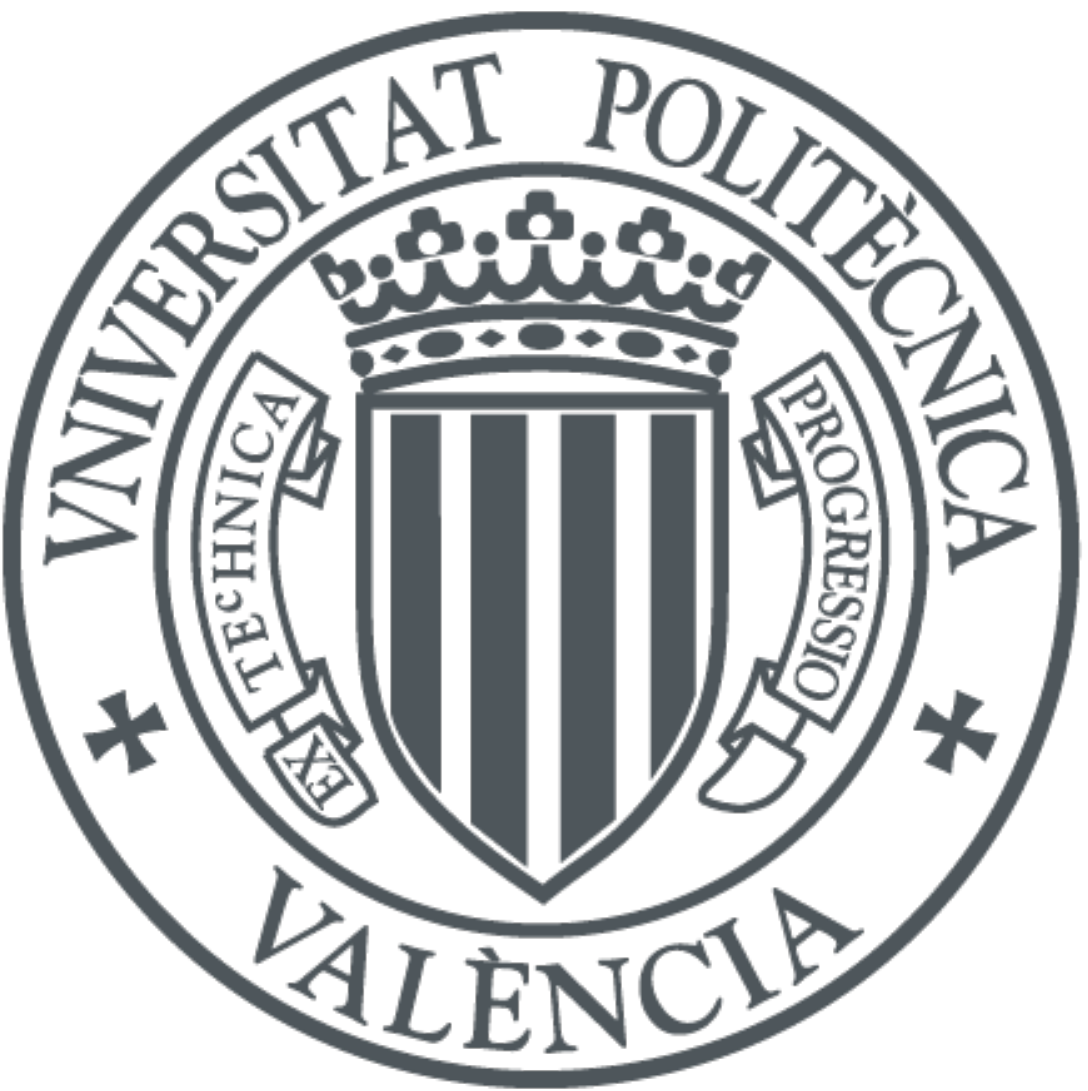

The final publication is available at

https://doi.org/10.1016/j.eurpolymj.2019.01.001

Copyright Elsevier

Additional Information 


\title{
DIELECTRIC SPECTROSCOPY OF NOVEL THIOL-ENE/EPOXY THERMOSETS OBTAINED FROM ALLYL-MODIFIED HYPERBRANCHED POLY(ETHYLENEIMINE) AND DIGLYCIDYLETHER OF BISPHENOL A
}

\author{
J.D. Badia ${ }^{1,2,3}$, R. Teruel-Juanes ${ }^{1}$, C. Acebo ${ }^{2}$, O. Gil-Castell ${ }^{1}$, A. Serra ${ }^{2}$, A. Ribes- \\ Greus ${ }^{1, *}$
}

${ }^{1}$ Instituto de Tecnología de Materiales (ITM), Universitat Politècnica de València (UPV). Camino de Vera s/n, 46022 Valencia, Spain.

${ }^{2}$ Departament de Química Orgànica i Analítica, Universitat Rovira i Virgili, C/ Marcel·lí Domingo s/n, 43007 Tarragona, Spain.

${ }^{3}$ Departament d'Enginyeria Química. Escola Tècnica Superior d'Enginyeria. Universitat de València. Av. de la Universitat, s/n, 46100, Burjassot, Spain.

*Corresponding author:

A. Ribes-Greus

aribes@ter.upv.es 


\title{
DIELECTRIC SPECTROSCOPY OF NOVEL THIOL-ENE/EPOXY THERMOSETS OBTAINED FROM ALLYL-MODIFIED HYPERBRANCHED POLY(ETHYLENEIMINE) AND DIGLYCIDYLETHER OF BISPHENOL A
}

\author{
J.D. Badia ${ }^{1,2,3}$, R. Teruel-Juanes ${ }^{1}$, C. Acebo ${ }^{2}$, O. Gil-Castell ${ }^{1}$, A. Serra ${ }^{2}$, A. Ribes-Greus ${ }^{1, *}$
}

Keywords: thermoset; epoxy resin; hyperbranched polymers; dielectric analysis; segmental cooperativity

\begin{abstract}
:
Dielectric Thermal Analysis (DETA) of a series of new thermoset obtained by click chemistry was performed. The new thermosets were obtained by a dual-curing process consisting in a first photochemical thiol-ene, followed by a thermal thiol-epoxy starting from an allyl-terminated hyperbranched poly(ethyleneimine) (HBPEI) and different proportions of diglycidylether of bisphenol A (DGEBA) and the corresponding stoichiometric proportions of pentaerythritol tetrakis (3-mercaptopropionate, PETMP). The dielectric behaviour was obtained experimentally supressing the conductive effects. Two sub-Tg intramolecular non-cooperative $\gamma$ and $\beta$ relaxations and an intermolecular cooperative $\alpha$-relaxation were detected. The $\gamma$-relaxation was ascribed to the dipole orientation of the terminal thiol groups. Two $\gamma$ relaxations $\gamma_{\text {epo }}$ and $\gamma_{\text {ene }}$ were observed, according to the influence of the surroundings. Only thermosets with a content of flexible HBPEI/PETMP domains higher than a $50 \% \mathrm{w} / \mathrm{w}$ showed the $\gamma_{\text {ene }}$ relaxation at lower temperatures and higher frequencies. The $\beta$-relaxation was a non-Johari-Goldstein relaxation, ascribed to the dipole transfer of the linear $-\mathrm{CH}_{2}-\mathrm{O}-(\mathrm{C}=\mathrm{O})-\mathrm{CH}_{2}-\mathrm{CH}_{2}$ - groups of the thiol constrained in the glassy state. The $\alpha$-relaxation was ascribed to the longterm segmental movements of the thermosets, occurring during the transition from the glassy to the rubbery stage. A synergic effect of reducing the rigidity and compactness of the thermosets by the reduction of aromatic moieties of the DGEBA/PETMP units, and the increase of the flexibility induced by the addition of HBPEI/PETMP units affected the macromolecular movement of the thermosets.
\end{abstract}




\section{Introduction}

Click reactions are extensively used in the preparation of materials since they can be performed in air and even in humid atmospheres, they are quantitative without sidereactions and therefore they lead to highly homogeneous networks. These reactions can be combined, since they are orthogonal to each other and therefore they are very adequate for dual-curing processes [1]. Click chemistry can be used for obtaining thermosets based on thiol chemistry. Under this framework, thiol-ene and thiol-epoxy reactions can be combined to offer novel materials, which cure under a two-step photo-thermal- dual process [2], which offer the best features of every family of materials. The controlled dual curing consisting in thiol-ene/thiol-epoxy reactions was stablished for the first time by Guzmán et al. [3]. The control of this process is based in the use of a latent curing amine compound that on heating liberates the amine and activates the second step of the curing process, which is the amine catalysed thiol-epoxy reaction.

This curing system was applied to the preparation of a series of new thermosets based in different proportions of allyl functionalized hyperbranched poly(ethyleneimine) (HBPEI) (see Figure 1), as -ene component and diglycidylether of Bisphenol A (DGEBA) as epoxy component, and stoichiometric amounts of pentaerythritol tetrakis (3mercaptopropionate) (PETMP) which acts as curing agent in both chemical processes [4]. It should be noticed that the use of hyperbranched polymers in thermosets have been extensively applied to enhance mechanical performance, especially toughness [5], [6]. From the point of view of the network structure, click reactions produce highly homogeneous networks whereas hyperbranched structures introduce heterogeneity in the material. It was reported that the higher the proportion of HBPEI in the materials, the lower the glass transition temperature $T g$, because of the flexibility introduced [6].. According to these characteristics, it seems interesting to go deeply in the study of these dual-cured networked structures. 


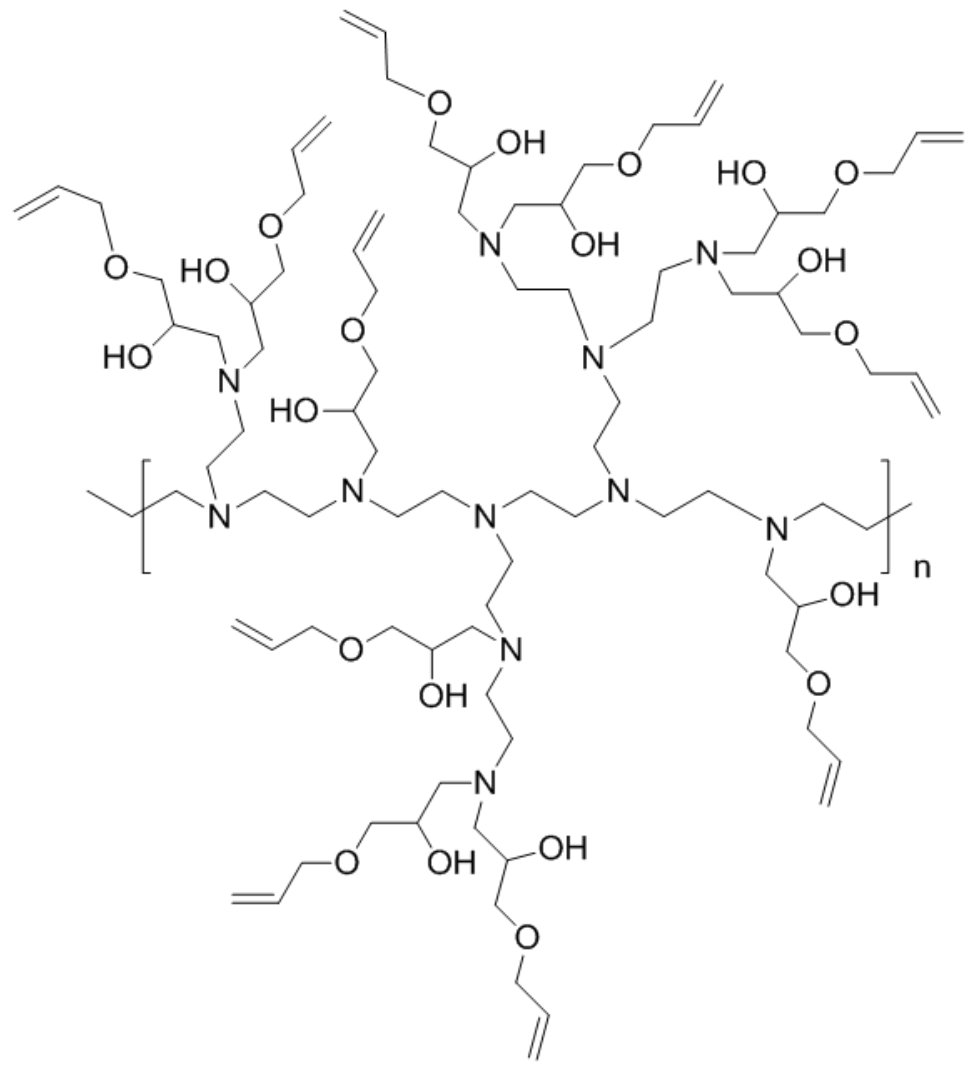

Figure 1. Idealized structure of HBPEI used as -ene multifunctional componente

Dielectric thermal broadband spectroscopy (DETA) is a highly versatile technique which can not only assess the conductive and isolating performance of materials, but can also help understand the macromolecular dynamics of the segmental movements of polymers [7]. The cooperativity of the movements of polarised groups can be intramolecular when it comes to the local movements of side groups to adapt to the electric field of the DETA. In contrast, the intermolecular movements imply the transmission of polarisation at large scale, usually related to movements of the polymer backbones. The study of the thermal activation of these movements can be of interest to tune the design of new thermosets.

The dielectric spectroscopy of thermosets applied to the epoxy systems based on diglycidylether of bisphenol A (DGEBA) [8]-[10], DGEBA cured with amines [11][14] with poly(ethyleneimine) [15], or in the presence of elastomers [16], [17] have been reported in the literature. The dielectrics of multiarm stars with nucleus of poly(etyleneimine) and arms of poly(lactide) alone [18] or dispersed in epoxy matrices[15], [19] have been also studied.

In this study, the DETA analysis of a thermoset, obtained by a dual photo/thermally curing, starting from mixtures in different proportions of an allyl-terminated 
hyperbranched poly(ethyleneimine) (HBPEI) and diglycidylether of bisphenol A (DGEBA) with the corresponding stoichiometric proportions of the tetrathiol pentaerythritol tetrakis (3-mercaptopropionate) (PETMP) was performed. The curing process consists of a first stage initiated by photoirradiation, which is a thiol-ene reaction between HBPEI and PETMP, which proceeds at room temperature. Thiol-ene reaction proceeds by the radical addition of sulphur radicals to the less hindered position of the allyl double bond, leading to linear moieties. This radical process is catalysed by a photoinitiator, in the present case DMAP (2,2-dimethoxy-2-phenylacetophenone). The structure originated by this process is represented in the upper part of Figure 2. The second stage of the curing occurs at higher temperature $\left(100^{\circ} \mathrm{C}\right)$ and consist in nucleophilic attack of the thiolate to the less substituted carbon of the oxirane ring, present in the DGEBA structure. Thiolate is produced by the presence of an amine, in this case 1-methylimidazole, which acts as the catalyst. The structure formed by this process is represented in the lower part of Figure 2. Both structures form a unique copolymeric network, with proportions depending on the relative amount of HBPEI and DGEBA. The participation of PETMP in both reactive processes and its multifunctionality link covalently both structures [4].

According to the above described precedents, the study by DETA of the thiol-epoxy/thiolene dual-cured materials represents an important contribution to the current state of the art, since to our understanding, this is the first report on the DETA study of this type of complex materials. The influence of the composition of the thermosets on the dielectric permittivity and on the segmental dynamics have been analysed. The cooperativity of movements and thermal activations of dielectric relaxations have been associated to the impact of the relative balance of thiol-epoxy DGEBA/PETMP and thiol-ene HBPEI/PETMP domains, which offer new perspectives to approach the design of future design of thermosets for specific mobility. 


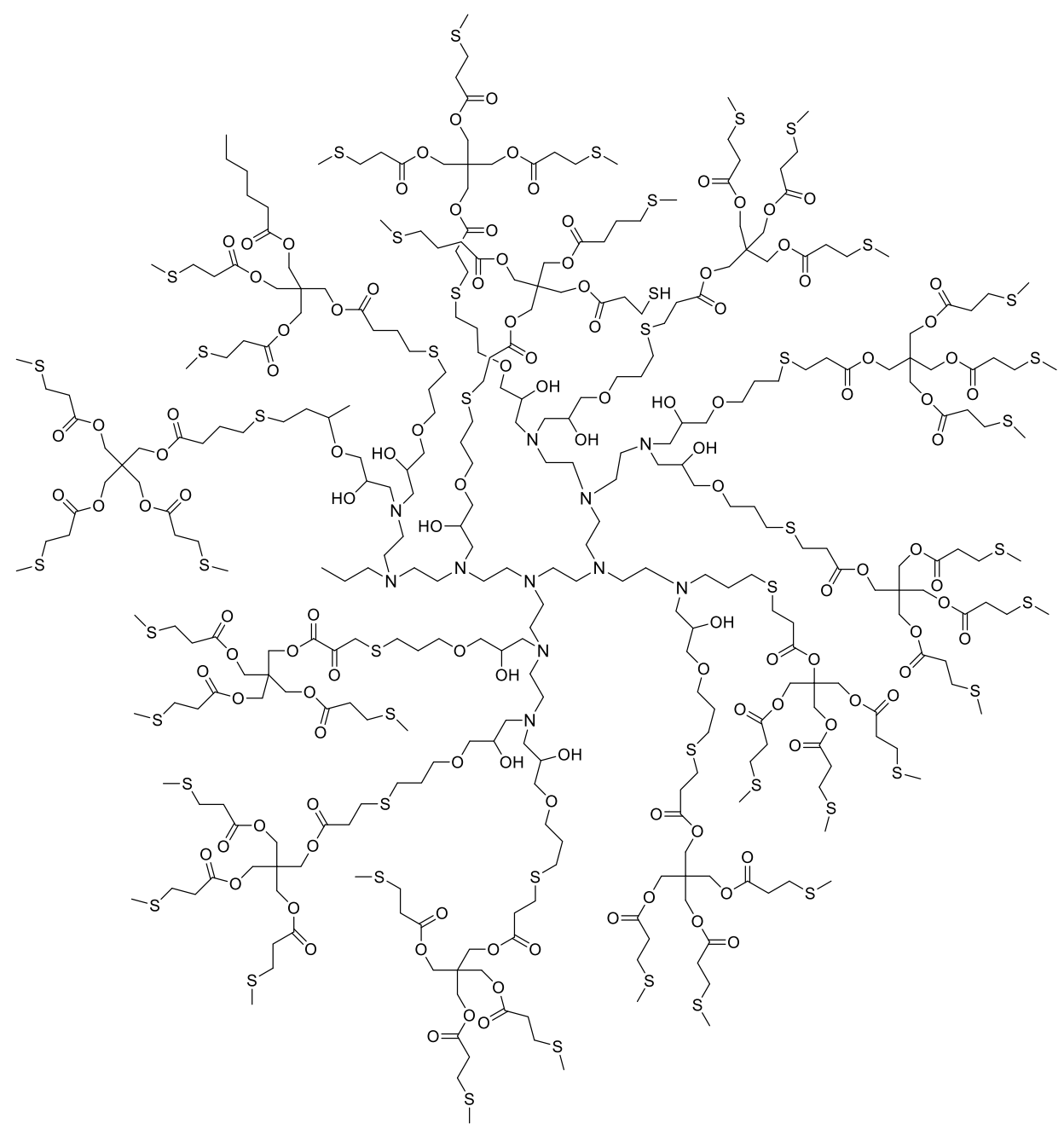

a) Thiol-ene network

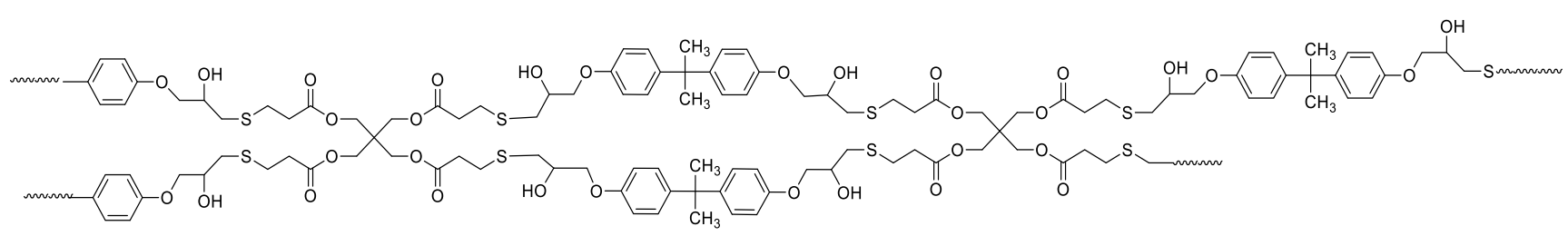

b) Thiol-epoxy

Figure 2. Idealized structures of both type of chemical structures formed in the copolymeric network a) Thiol-ene network formed by photochemical reaction of HBPEI with PETMP and b) Thiol-epoxy network by thermal reaction of DGEBA with PETMP. Thiol monomer act as a link between both network structures. 


\section{Experimental procedure and calculations}

\subsection{Materials and reagents}

Poly(ethyleneimine) (PEI) Lupasol®FG ( $800 \mathrm{~g} / \mathrm{mol})$ was kindly donated by BASF and used without further purification. From the molecular weight of the polymer and of the repeating unit an average degree of polymerization of 18.6 was calculated. According to the data sheet, the relationship $\left(\mathrm{NH}_{2} / \mathrm{NH} / \mathrm{N}\right)$ was $(1 / 0.82 / 0.53)$ and thus by calculations the equivalent number of primary, secondary and tertiary amines resulted to be 0.010 , 0.00837, and $0.0053 \mathrm{eq} \cdot \mathrm{g}^{-1}$. Allyl glycidyl ether, pentaerythritol tetrakis (3mercaptopropionate) (PETMP), 1-methylimidazole (1-MI) and 2,2-dimethoxy-2phenylacetophenone (DMPA) were all purchased from Sigma-Aldrich. Isopropanol was purchased from Scharlab. Diglycidylether of bisphenol A (DGEBA) Araldite GY 240 $(\mathrm{EEW}=182 \mathrm{~g} / \mathrm{eq})$ was provided by Huntsman.

The synthesis of allyl-terminated hyperbranched poly(ethylenimine) (HBPEI) was performed by reacting PEI with allyl glycidyl ether at $50^{\circ} \mathrm{C}$ in isopropanol, as previously described [4].

\subsection{Preparation of the dual-cured thermosets}

The samples were prepared starting from their corresponding mixtures by irradiation under light-curing equipment (Dymax ECE 2000 UV light-curing flood lamp system) during $2 \mathrm{~min}$ (in intervals of $30 \mathrm{~s}$ with $5 \mathrm{~min}$ among them, $1 \mathrm{~min}$ for each face of the sample) (UV-intensity of $105 \mathrm{~mW} \cdot \mathrm{cm}^{-2}, 365 \mathrm{~nm}$ ) and then thermally treated at $100^{\circ} \mathrm{C}$ for $1 \mathrm{~h}$ and $120{ }^{\circ} \mathrm{C}$ for $30 \mathrm{~min}$ in an oven, as previously described [4]. A series of thermosets with different compositions were prepared by changing the proportion of HBPEI and DGEBA. PETMP was added in stoichiometric proportions to the reactive groups. $1 \mathrm{phr}$ (parts per hundred parts of reactive mixture) of DMAP was added to the formulations cured by irradiation and $2 \mathrm{phr}$ of 1-MI were added to the thermally-cured formulations. The thermosets were labelled according to their composition, as follows: DGEBA/PETMP and HBPEI/PETMP for the 100\% thiol-epoxy and thiol-ene thermosets, respectively, and DGEBA-XXHBPEI/PETMP, for the dual-cured thermosets with a XX of 25,50 and $75 \%$ w/w of thiol-ene content in the final material. 


\subsection{Dielectric Spectroscopy}

The dielectric spectra (DS) of the samples were obtained using an Alpha mainframe frequency analyser in conjunction with an active cell (Concept 40, Novocontrol Technologies BmgH \& Co. Kc, Hundsangen, Germany). In order to supress the usual conductive effects, the sample electrode assembly consisted in the sample and a Teflon ${ }^{\mathrm{TM}}$ film inserted as blocking layer between the sample and one electrode [20]. The sample/Teflon sandwich is a Maxwell-Wagner-Sillars [21]-[23] layer capacitor, which suppresses the direct current and avoids at low temperatures the dipole motion and the charge accumulation at the electrode interface [24]-[26]. The response was measured under isothermal conditions in the frequency range $\mathrm{f}=10^{-2}-10^{6} \mathrm{~Hz}$, at temperatures of $150^{\circ} \mathrm{C}$ to $80^{\circ} \mathrm{C}$, controlled by the Quatro system (Novocontrol Technologies, Germany) by increasing steps of $10^{\circ} \mathrm{C}$ under inert nitrogen atmosphere and using liquid nitrogen as coolant.

The methodology of analysis which involves the phenomenological study of the dielectric permittivity and dielectric loss, along with the thermal activation of each dielectric relaxation can be found elsewhere [27]-[30]. Shortly, the complex dielectric permeability, $\varepsilon^{*}=\varepsilon^{\prime}-i \cdot \varepsilon^{\prime}$ ' was gathered, being $\varepsilon^{\prime}$ and $\varepsilon^{\prime}$ ' their real and imaginary parts of the dielectric permittivity, and $\tan (\delta)=\varepsilon^{\prime} / \varepsilon$ ' the trigonometric tangential relationship between both terms, related to the phase angle $\delta$. The $\varepsilon^{*}$ curves were deconvoluted by means of the Charlesworth [31] method and modeled by superimposing HavriliakNegami (HN) functions (Eq. 1) [32], [33] , where $\alpha_{H N}$ and $\beta_{H N}$ are parameters corresponding to the width and asymmetry of the relaxation time distributions, respectively; $\tau_{H N}$ is the Havriliak-Negami relaxation time and $\Delta \varepsilon=\varepsilon_{S}-\varepsilon_{\infty}$ the dielectric strength (with $\varepsilon_{S}$ and $\varepsilon_{\infty}$ are the real part of the permittivity when the angular frequencies are $\omega \rightarrow 0$ and $\omega \rightarrow \infty$, respectively). The sub index $k$ represents the number of the individual HN contributions, if any. Finally, the relaxation times and frequencies $\left(f_{\max }=\right.$ $\left.1 / \tau_{\max }\right)$ of the HN individual $\varepsilon^{*}$ curves were calculated according to $\boldsymbol{E q} .2$.

In order to discriminate cooperative from non-cooperative segmental movements, the temperature dependence of the relaxation times was analysed by means of the Eyring model [34], [35]. Non-cooperative relaxations, due to the independent motion of kinetic units, follow a zero entropy law, and the relaxations lie within the line represented by 
$E a=R T^{\prime}\left[1+\ln (k / 2 p h)+\ln T^{\prime}\right]=R T^{\prime}\left[22.92+\ln T^{\prime}\right](\boldsymbol{E q} .3)$, where $T^{\prime}$ is the temperature at which the frequency of the corresponding relaxation is $f=1 \mathrm{~Hz}[36]$.

The thermal activation of the dielectric phenomena was characterised by Arrhenius maps which plot $\log f_{\max }$ versus the inverse of temperature, being $f_{\max }$ the maximum frequency of the relaxations at each isotherm. Intramolecular non-cooperative relaxations usually display Arrhenius-like linear relationships $f_{\max }(T)=f_{0} \cdot \exp (-E a / R \cdot T)(\boldsymbol{E q} \cdot 4)$, while intermolecular cooperative relaxations show a curvature at high frequencies and low temperatures, and therefore a modification of the Arrhenius model is more appropriate.

The empirical Vogel-Fulcher-Tamman-Hesse model [37], [38] is $f_{\max }(T)=f_{0} \cdot \exp [-B / R \cdot(T-$ $\left.\left.T_{K}\right)\right]$. In these equations $B=D \cdot T_{K}(\boldsymbol{E q} 5), E a$ is the apparent activation energy, $f_{0}$ is a preexponential term, $\tau$ is the relaxation time (s), that is $(2 \cdot \pi \cdot f)^{-1}, \mathrm{R}$ is the universal constant for ideal gases $\left(8.31 \mathrm{~J} \cdot \mathrm{mol}^{-1} \cdot \mathrm{K}^{-1}\right), B(\mathrm{~K})$ and $T_{K}(\mathrm{~K})$ are positive parameters specific to the material, and $D$ is a non-dimensional factor termed as fragility or strength parameter. $T_{K}$ typically appears $40-60 \mathrm{~K}$ below the glass transition temperature $T_{g}$. In addition, the fragility index $m$ permits an assessment of the deviation of $\tau(T)$ from the Arrhenius behavior of polymers [39]. Calculated at the $T g, m=(B \cdot T g) \cdot\left[\ln (10)^{-1} \cdot\left(T g-T_{K}\right)^{-2}\right](E \boldsymbol{E}$. 6). Finally, the apparent activation energy of intermolecular cooperative relaxations can be calculated at the $T g$ as $E a=\left(R \cdot B \cdot\left[1-\left(T_{K} / T g\right)^{2}\right]^{-1}(\boldsymbol{E} \boldsymbol{q} 7)[7]\right.$.

$$
\begin{gathered}
\varepsilon^{*}-\varepsilon_{\infty}=\sum_{k}\left[\frac{\Delta \varepsilon}{\left\{1+\left(i w \tau_{H N k}\right)^{\alpha_{k}}\right\}^{\beta_{k}}}\right] \\
\tau_{\max }=\tau_{H N}\left[\frac{\sin \left(\frac{\pi\left(\alpha_{H N}\right) \beta_{H N}}{2\left(\beta_{H N}+1\right)}\right)}{\sin \left(\frac{\pi\left(\alpha_{H N}\right)}{2\left(\beta_{H N}+1\right)}\right)}\right]^{1 / \alpha_{H N}}
\end{gathered}
$$




\section{Results and discussion}

Dielectric Thermal Analysis (DETA) is of great importance to test the segmental dynamics of polymers [27], [30], [40]-[42], whose modifications are not perceptible by other macroscopic techniques. The dielectric spectrum was measured by experimentally removing the term of the conductivity [20], in contrast to other DETA analyses reported for DGEBA-based and hyperbranched poly(ethylenimine)-based thermosets, which usually do not suppress them or consider mathematical deconvolutions. The analysis was conducted essentially through the loss tangent $(\tan \delta)$ and the complex dielectric permeability $\varepsilon^{*}=\varepsilon^{\prime}-i \varepsilon^{\prime}$, taking into account the real $\left(\varepsilon^{\prime}\right)$ and imaginary ( $\left.\varepsilon^{\prime \prime}\right)$ parts. The 3-dimensional plot of the dielectric tan $\delta$ of DGEBA/PETMP, HBPEI/PETMP and their thiol-epoxy/thiol-ene thermosets in a large range of frequency and temperature are shown in Figure 3. In addition, the isochrones of the $\tan \delta$ are shown in Figure 4. Apparently, in the increasing temperature order, the dielectric spectra unveiled a low-intensity relaxation with peaks between -150 and $60{ }^{\circ} \mathrm{C}$, followed by an intermediate-intensity relaxation with peaks between $-90^{\circ} \mathrm{C}$ and $20^{\circ} \mathrm{C}$ and a final relaxation with high intensity and peaks between 10 and $80^{\circ} \mathrm{C}$. These peaks will be lately ascribed as two $\gamma$ and $\beta$ relaxations in the glassy state, followed by the $\alpha$ relaxation during the glass-rubber relaxation, respectively. As expected, higher frequencies shifted the dielectric signals to higher temperatures. 

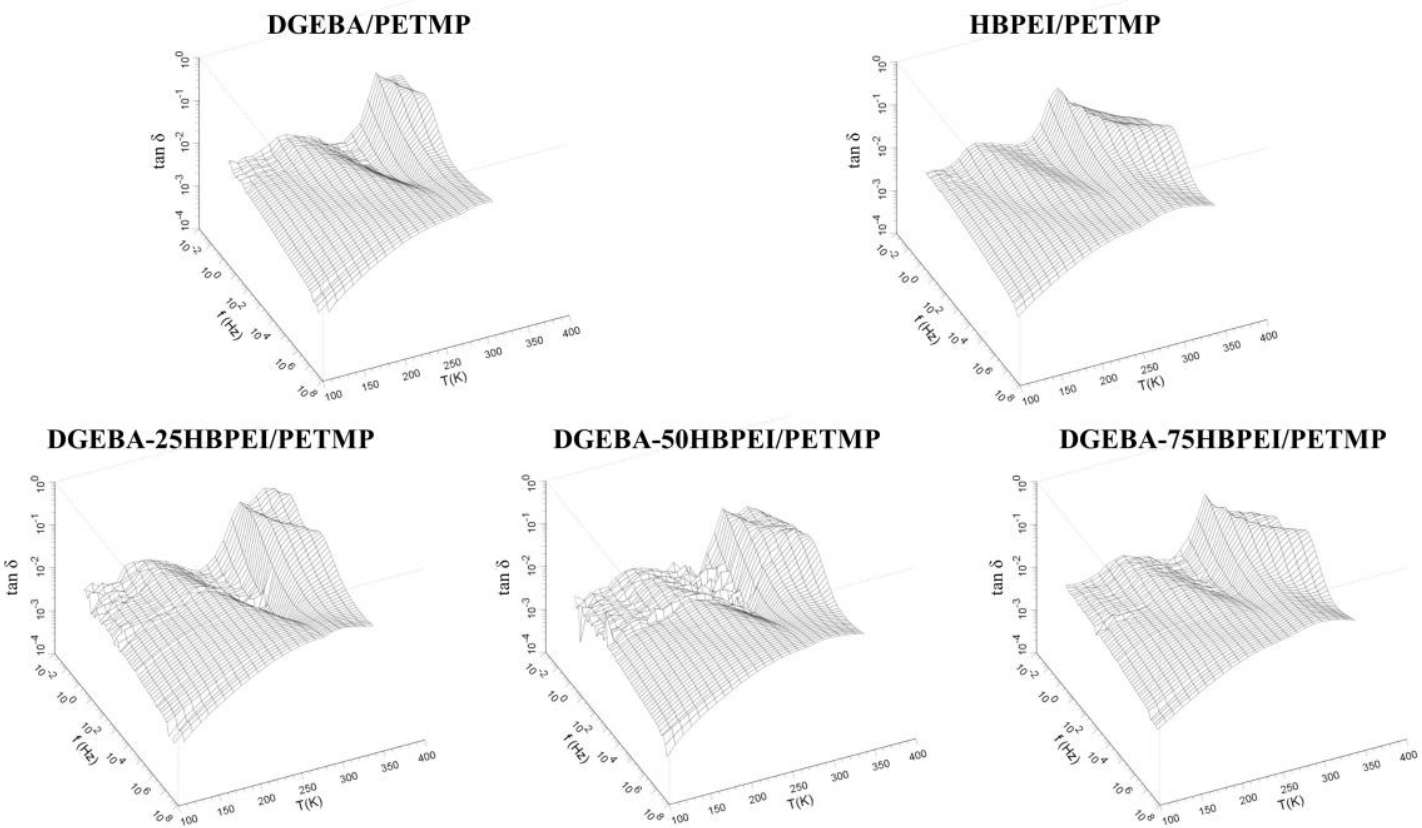

Figure 3. 3D plot of the dielectric loss tan $\delta$ of DGEBA/PETMP, HBPEI/PETMP and their thiolepoxy/thiol-ene thermosets 

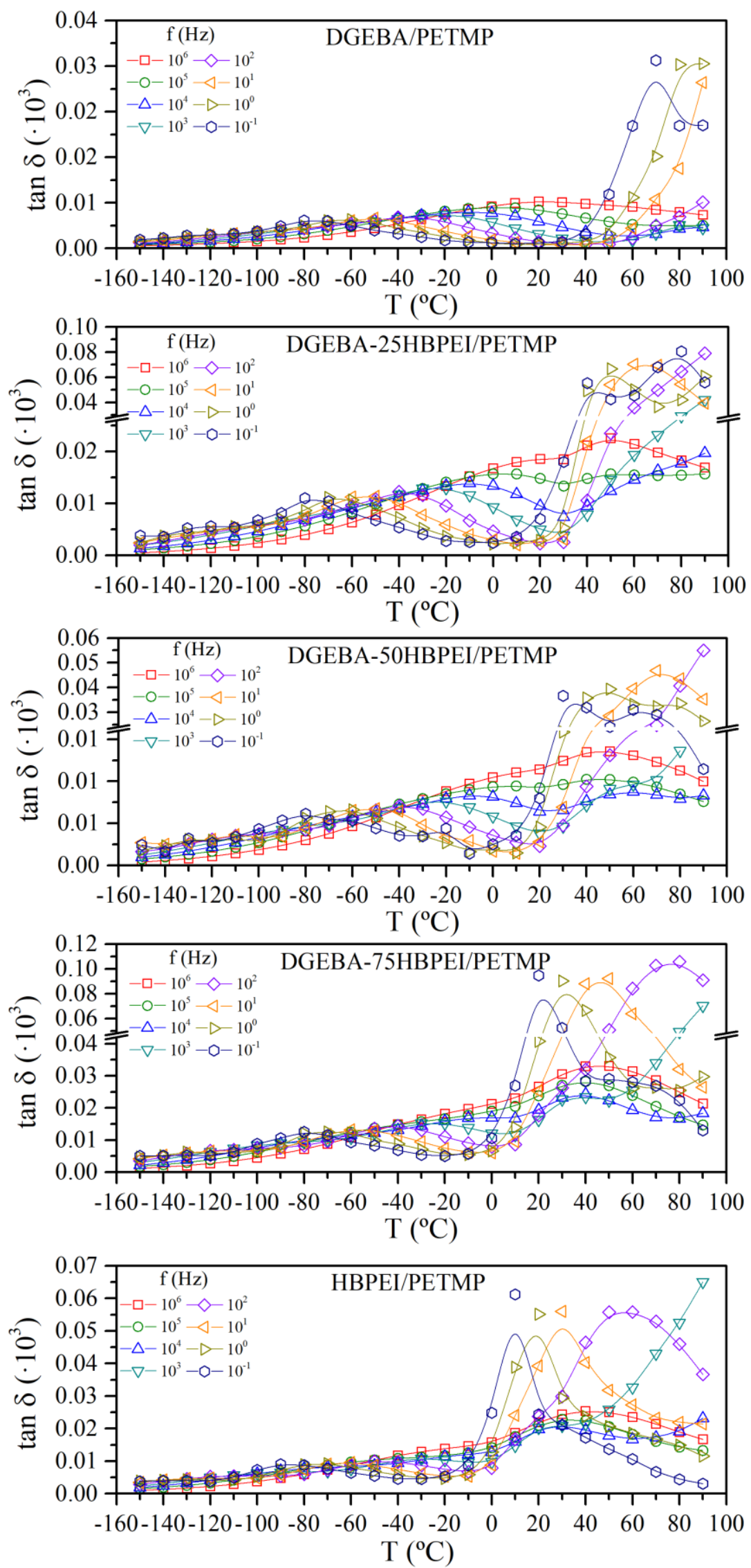

Figure 4. Thermal evolution of the loss tan $\delta$ of DGEBA/PETMP, HBPEI/PETMP and their thiol epoxy/thiol-ene thermosets. 
The plots corresponding to the frequency-response of the dielectric permittivity $\varepsilon$ ' are shown in Figure 5 for DGEBA/PETMP, HBPEI/PETMP and their thiol-epoxy/thiol-ene thermosets for negative and positive temperature ranges. The remarkable jump of dielectric permittivity at a certain temperature for each thermoset could be indicative of surpassing the glass transition, since the polarisation would be more significant the lower the macromolecular constraints of the thermoset were. The presence of HBPEI/PETMP domains in the thermoset would imply a lower glass transition temperature and a higher flexibility. These hypothesis shall be confirmed by the study of the thermal activation dynamics and segmental cooperativity of the dielectric relaxations, as shown in the next section. 

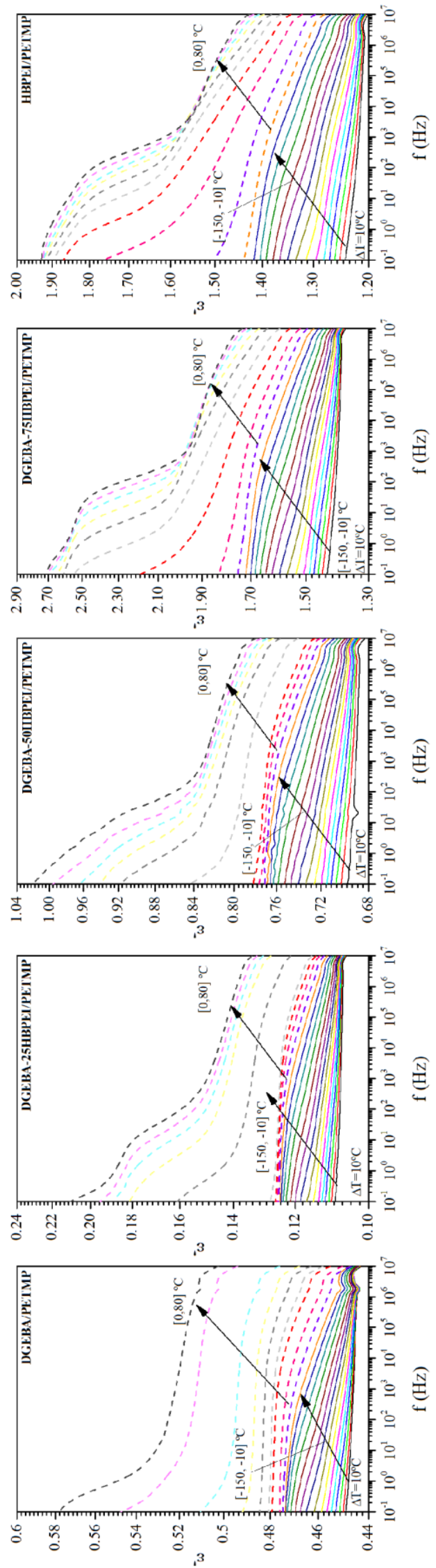

Figure 5. Frequency-dependence of the isotherms of the dielectric permittivity of DGEBA/PETMP, HBPEI/PETMP and their thiol-epoxy/thiol-ene thermosets. Solid lines represent negative temperatures, while dashed lines show positive temperatures, in the Celsius scale 


\subsection{Segmental cooperativity of dielectric relaxations of DGEBA/T, HBPEI/T and their}

thiol-epoxy/thiol-ene thermosets

The Eyring plot for DGEBA/PETMP, HBPEI/PETMP and their thiol-epoxy/thiol-ene thermosets is shown in Figure 6. Three main groups of dielectric relaxations can be pointed out. In the increasing order of temperatures, a $\gamma$-like relaxation was firstly found, which lie close to the $\Delta S=0$ line, which is a sign to ascribe intramolecular non-cooperative origin to the dipole movement. Later on, a $\beta$-like relaxation, also close to the $\Delta S=0$ line, but with a slight higher distance than that of the $\gamma$ relaxation was shown. Despite it might be more affected by the macromolecular movements activated by a higher temperature, it could also be explained in terms of intramolecular non-cooperative dipole transfer mechanisms. Finally, at high temperatures, an $\alpha$-like relaxation deviates from the linearity of $\Delta S=0$, and therefore it may be explained in terms of intermolecular cooperative dipole transfer occurring during the long-term segmental movements of the thermosets. The results are in agreement with those reported for neat DGEBA thermosets, which showed a primary $\alpha$-like relaxation and two secondary $\gamma$ and $\beta$ relaxations [10], [11]. They are as well in consonance with the studies of molecular mobility of hyperbranched polymers included in epoxy matrices in thermally-cured thermosets, related by Roman et al [15]. The specific ascription of each dielectric relaxation to the molecular structure of the thermosets and the discrimination between cooperative and non-cooperative movements will be shown hereinafter, along with the analysis of their thermal activations. 


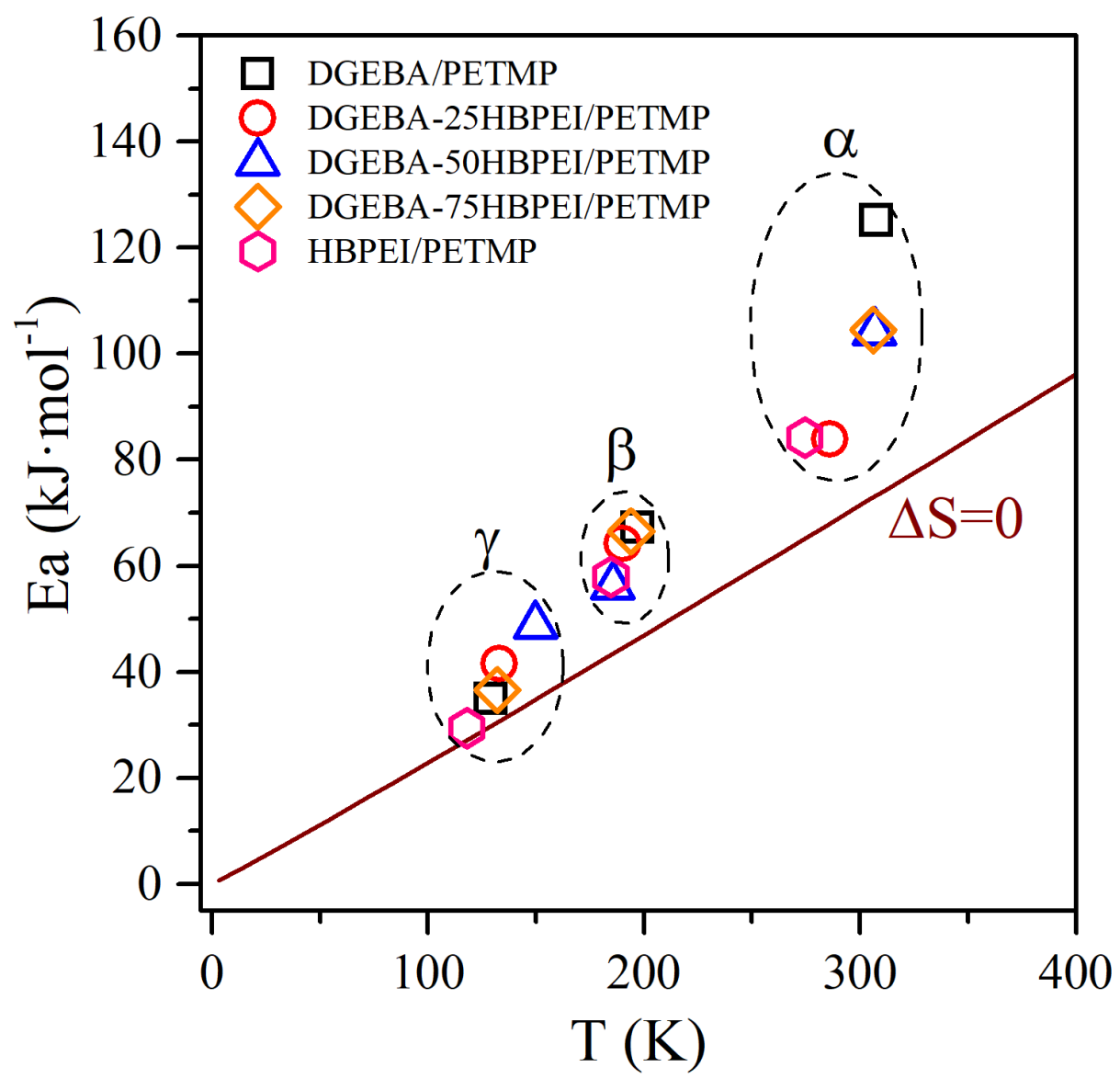

Figure 6. Eyring plot for DGEBA/PETMP, HBPEI/PETMP and their thiol-epoxy/thiol-ene thermosets.

The Arrhenius plots of DGEBA/PETMP, HBPEI/PETMP and the dual cured thermosets are shown in Figure 7. The following observations can be drawn, in the increasing temperature order: two intramolecular non-cooperative $\gamma$ and $\beta$ relaxations and one structural intermolecular cooperative $\alpha$ relaxation, in agreement with the observations raised by the analysis of the Eyring plot, and based on the structural arguments discussed hereinafter. 


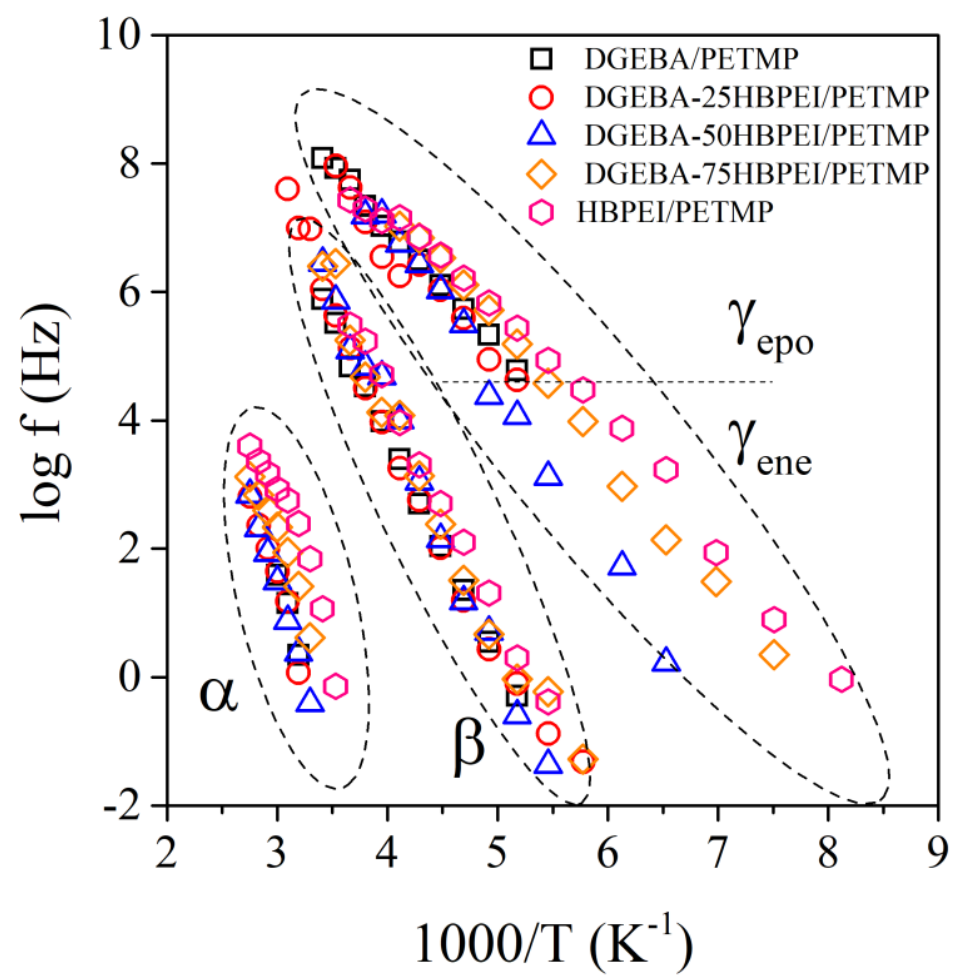

Figure 7. Arrhenius plot for DGEBA/PETMP, HBPEI/PETMP and their thiol-epoxy/thiol-ene thermosets

\subsection{Analysis of thermal activations of each dielectric relaxation}

\subsubsection{Analysis of the intramolecular non-cooperative $\gamma$-relaxations}

Two $\gamma$-like dielectric relaxations appeared in a range of temperatures of $\sim[-150,-90]{ }^{\circ} \mathrm{C}$ and frequencies of $\sim\left[10^{0}, 10^{8}\right] \mathrm{Hz}$, as shown in Figure 7. They were labelled as $\gamma_{\text {epo }}$ or $\gamma_{\text {ene }}$, which subscripts relate to o the predominant phase of DGEBA/PETMP (epoxy) or HBPEI/PETMP (ene) present in the thermoset. On the one hand, thermosets with higher DGEBA/PETMP content (DGEBA, and DGEBA-25HBPEI/PETMP) showed the $\gamma_{\text {epo }}$ relaxation in a range of temperatures of $\sim[-20,60]^{\circ} \mathrm{C}$ and frequencies of $\sim\left[10^{0}, 10^{4}\right] \mathrm{Hz}$. On the other hand, thermosets with higher HBPEI/PETMP domains in the composition (DGEBA-50HBPEI/PETMP, DGEBA-75HBPEI/PETMP, HBPEI/PETMP) showed the $\gamma_{\text {ene }}$ relaxation in a range of temperatures of $\sim[-150,-20]{ }^{\circ} \mathrm{C}$ and frequencies of $\sim\left[10^{4}, 10^{8}\right]$ $\mathrm{Hz}$. 
The linear behaviour of $\log \left(f_{\max }\right)$ vs $T^{-1}$ of the $\gamma$-relaxations, i.e. $\gamma_{\text {epo }}$ and $\gamma_{\text {ene }}$, can be explained by means of Arrhenius-like functions, by means of Eq 4, where the Ea stands for the apparent activation energy. All results are gathered in Table 1, with correlation fitting values between 0.95 and 0.99 . The assignation of the $\gamma$-relaxation to intramolecular movements of the terminal units of the hyperbranched polymers also corroborates the discussions drawn from the analysis of the Eyring plot. The $E a$ of this $\gamma_{e n e}$ relaxation is of the same order of that of $\gamma_{e p o}$, with a confidence correlation fitting coefficient between 0.98 and 0.99 , thus confirming the hypothesis of similar molecular origin. Both relaxations can be ascribed to the common terminal thiol groups, but those with more flexible HBPEI/PETMP units are more likely to transmit dipoles at lower temperatures and lower frequencies, since the steric constraints of the vicinities are reduced [15]. Neat DGEBA/PETMP and HBPEI/PETMP respectively showed $E a \sim 36$ and $27 \mathrm{~kJ} \cdot \mathrm{mol}^{-1}$, with narrow error margins. This difference may be ascribed to the higher volume per unit of functionality of HBPEI/PETMP, as well as to the lower compaction of the hyperbranched polymer [4]. The Ea obtained for the thermosets did not follow a composition rule. The relatively low $E a$ values are in agreement with those reported for the $\gamma$ relaxation for other hyperbranched thermosets based on polyglycerol, polyurethane, polyamide, polyester amides or polyetherimines [15], [43]-[47].

Table 1. Results of Arrhenius fitting to intramolecular non-cooperative relaxations of DGEBA/PETMP, HBPEI/PETMP and their thiol-epoxy/thiol-ene thermosets.

\begin{tabular}{|c|c|c|c|c|c|c|}
\hline Relaxation & Parameter & DGEBA/PETMP & $\begin{array}{c}\text { DGEBA- } \\
\text { 25HBPEI/PETMP }\end{array}$ & $\begin{array}{c}\text { DGEBA- } \\
\text { 50HBPEI/PETMP }\end{array}$ & $\begin{array}{c}\text { DGEBA- } \\
\text { 75HBPEI/PETMP }\end{array}$ & HBPEI/PETMP \\
\hline \multirow{2}{*}{$\gamma_{\text {epo }}$} & $\begin{array}{c}\text { Ea } \\
\left(\mathbf{k J} \cdot \mathbf{m o l}^{-1}\right) \\
\end{array}$ & $35,81 \pm 0.55$ & $36,60 \pm 2.67$ & $38,20 \pm 3.09$ & $35,98 \pm 0.97$ & $27,52 \pm 1.15$ \\
\hline & $\mathbf{R}^{2}$ & 0,997 & 0,954 & 0,968 & 0,994 & 0,982 \\
\hline \multirow{2}{*}{$\gamma_{\text {ene }}$} & $\begin{array}{c}\text { Ea } \\
\left(\mathbf{k J} \cdot \mathbf{m o l}^{-1}\right) \\
\end{array}$ & --- & --- & $43,44 \pm 3.49$ & $38,55 \pm 2.33$ & $38,37 \pm 1.70$ \\
\hline & $\mathbf{R}^{2}$ & --- & --- & 0,981 & 0,986 & 0,990 \\
\hline \multirow{2}{*}{$\beta$} & $\begin{array}{c}\text { Ea } \\
\left(\mathrm{kJ} \cdot \mathrm{mol}^{-1}\right) \\
\end{array}$ & $67,25 \pm 0.70$ & $66,49 \pm 2.21$ & $73,72 \pm 1.99$ & $64,17 \pm 2.76$ & $64,73 \pm 1.24$ \\
\hline & $\mathbf{R}^{2}$ & 0,998 & 0,983 & 0,992 & 0,978 & 0,996 \\
\hline
\end{tabular}




\subsubsection{Analysis of the intramolecular non-cooperative $\beta$-relaxations}

A $\beta$-like dielectric relaxation was displayed in a range of temperatures of $\sim[-90,20]^{\circ} \mathrm{C}$ and frequencies of $\sim\left[10^{-2}, 10^{6.5}\right] \mathrm{Hz}$, as shown in Figure 7. The linear behaviour of $\log$ $\left(f_{\max }\right)$ vs $T^{-1}$ of the $\beta$-relaxation can be also modelled in terms of Arrhenius-like functions (Eq. 4). The apparent activation energies, $E a$, are gathered in Table 1, with correlation fitting values between 0.97 and 0.99 .

Since the $\beta$-relaxation appears for both neat DGEBA/PETMP and HBPEI/PETMP thermosets, it can be therefore ascribed to the presence of the thiol curing agent. In particular, it can be explained by the dipole transfer of the linear $-\mathrm{CH}_{2}-\mathrm{O}-(\mathrm{C}=\mathrm{O})-\mathrm{CH}_{2}$ $\mathrm{CH}_{2-}$ groups confined in the supercooled / glassy state, where no relevant structural movements are allowed at large scale [15]. The $E a$ values were in the $\sim[64,73] \mathrm{kJ} \cdot \mathrm{mol}^{-1}$ range, which are higher than those of the $\gamma$ relaxation, and in agreement with those found for the $\beta$-relaxation of other hyperbranched thermosets [15], [43]-[47].

Traditionally, $\beta$ relaxations were considered to originate from rotations of side chains or functional groups of polymer. However, Johari and Goldstein [48] reported $\beta$ relaxations in several glasses of rigid molecules, suggesting that some $\beta$ relaxations also involve the motion of the entire macromolecule instead of intramolecular degrees of freedom, with a strong connection to the structural $\alpha$ relaxation and, therefore, involving high activation energies. Since the $\beta$ and the $\alpha$ relaxation seem not to merge even by extrapolation in Figure 7, it can be considered that the $\beta$ relaxation of the thiol- epoxy/ene thermosets was not a Johari-Goldstein (JG) relaxation, and therefore, both relaxations were not connected [49]. Therefore, the assignation of being an intramolecular non-cooperative relaxation during the analysis of the Eyring plot is strengthened. This is in contrast to the JG behaviour of the $\beta$ relaxation of other hyperbranched thermosets, such as polyglycerols and polyester amides [43], [47], but is in agreement with the non-JG behaviour of hyperbranched polymers based on polyesters or polyetherimines [15], [50]. 


\subsubsection{Analysis of the intermolecular cooperative $\alpha$-relaxation}

An $\alpha$-like dielectric relaxation occurred in a range of temperatures of $\sim[10,80]{ }^{\circ} \mathrm{C}$ and frequencies of $\sim\left[10^{0}, 10^{4}\right] \mathrm{Hz}$, as shown in Figure 7. The effects of the progressive replacement of thiol-epoxy DGEBA/PETMP units by thiol-ene HBPEI/PETMP units is shown in the detail of the Arrhenius map for the $\alpha$ dielectric relaxation of Figure 8. From pure DGEBA/PETMP, its substitution by HBPEI/PETMP units up to a 50\%, enlarges the temperature and frequency ranges of the relaxation, but still following a similar thermalactivation behaviour, since all new frequency-temperature coordinates of the DGEBA/PETMP, DGEBA-25HBPEI/PETMP and DGEBA-50HBPEI/PETMP thermosets seem to closely lie on the line of extrapolation of that of the DGEBA/PETMP. In contrast, the thermosets of DGEBA-75HBPEI/PETMP and HBPEI/PETMP showed a progressive displacement of the dielectric relaxations towards lower temperatures. In addition, the relaxations happened in a wider temperature range towards lower temperatures and a wider frequency ranges towards higher frequencies.

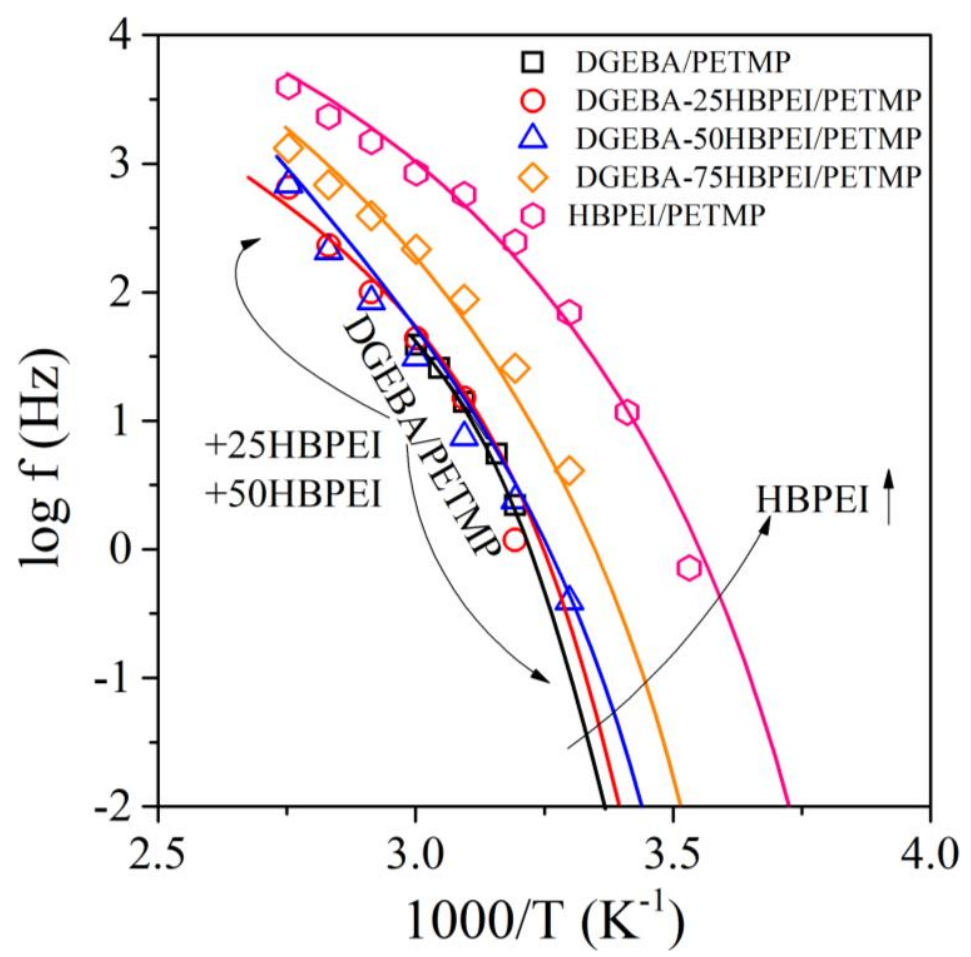

Figure 8. Detail of Arrhenius plot for the $\alpha$-relaxation of DGEBA/PETMP, HBPEI/PETMP and their thiol-epoxy/thiol-ene thermosets.

The non-linear behaviour of $\log \left(f_{\max }\right)$ vs $T^{-1}$ of the $\alpha$-relaxation can be modelled in terms of Vogel-Flucher-Tamman-Hesse (VFTH)-like functions (Eq. 5), which is an empirical 
equation that assumes a correction of the Arrhenius model to take into account the curvature of the frequency-temperature relationship at high frequencies and high temperatures. The non-linear behaviour indicates that the $\alpha$-relaxation can be understood as an intermolecular cooperative relaxation, in agreement with the discussions of the Eyring plot. This relaxation was therefore ascribed to the long-term segmental movements of the thermosets occurring during the transition from the glassy to the rubbery stage. The results of the thermal activation parameters as $D, B$ and $m$, obtained for the best fit of the VFTH equation, showed that the thiol-epoxy/thiol-ene thermosets can be classified as strong glass-formers [39], [51]. The values of $m$ lie within the linear relationship proposed by Qin and McKenna for polymers [52], where $m(T g)=0.28( \pm 0.067) \cdot T g(K)+9( \pm 20)(\boldsymbol{E q}$. 8). The small differences reported for the dynamic fragility parameters do not highlight significant composition-based discussions. Nonetheless, one should take into account that the frequency/temperature range of the $\alpha$-relaxation might not be representative enough to establish a general discussion about these parameters to understand the influence of the relative contribution of each domain in the thermoset [15]. In this sense, the differences of the apparent activation energy $E a$, calculated by $E q$. 7 were more relevant. Since the $E a$ depends on the temperature chosen for the analysis, in order to obtain results comparable with the ones of the literature, a convention was followed. The Ea was reported for the dielectrometric glass transition temperature, i.e, the temperature at which the relaxation time is $100 \mathrm{~s}$, as shown in Table

2. The values were gathered in the $\sim[155,255] \mathrm{kJ} \cdot \mathrm{mol}^{-1}$ range, from neat HBPEI/PETMP to neat DGEBA/PETMP thermosets. These values are slightly higher than those reported for thermally-cured HBPEI/PETMP and slightly lower than those reported by DGEBA/PETMP thermosets [15], but similar to those shown for other hyperbranched polymers such as polyamide amines [53] or polyester amides [47], calculated at the $T g$ after reconsideration of VFTH analysis by Roman et al [15]. They are also in agreement with binary thermosets based on DGEBA and HBPEI [15]. In any case, the $E a$ values were remarkably higher than those obtained for the $\beta$ relaxations, which reveal a higher order of segmental dynamics implied during the dielectric relaxation, which is consistent with the arguments of cooperativeness drawn during the assignation of macromolecular movements. 
Table 2. Analysis of the intermolecular cooperative $\alpha$-relaxation of DGEBA/PETMP, HBPEI/PETMP and their thiol-epoxy/thiol-ene thermosets.

\begin{tabular}{|c|c|c|c|c|c|c|}
\hline & & DGEBA/PETMP & $\begin{array}{c}\text { DGEBA- } \\
\text { 25HBPEI/PETMP }\end{array}$ & $\begin{array}{c}\text { DGEBA- } \\
\text { 50HBPEI/PETMP }\end{array}$ & $\begin{array}{c}\text { DGEBA- } \\
\text { 75HBPEI/PETMP }\end{array}$ & НBPEI/PETMP \\
\hline \multirow{2}{*}{$\mathrm{Tg}$} & ${ }^{*} \operatorname{Tg}\left({ }^{\circ} \mathrm{C}\right)$ & 23,85 & 21,49 & 17,72 & 11,35 & $-4,62$ \\
\hline & ${ }^{* *} \Delta \operatorname{Tg}(\%)$ & 0 & 8 & 22 & 44 & 100 \\
\hline \multirow{4}{*}{ VFTH fit } & $\operatorname{lnf}_{0}(\mathrm{~Hz})$ & $13,35 \pm 0.32$ & $14,51 \pm 0.68$ & $13,59 \pm 0,53$ & $13,62 \pm 0,27$ & $15,21 \pm 0,52$ \\
\hline & D & $7,70 \pm 0,21$ & $9,05 \pm 0,56$ & $8,79 \pm 0,43$ & $8,18 \pm 0,25$ & $10,16 \pm 0,52$ \\
\hline & ${ }^{* * * *} \mathbf{T}_{\mathbf{K}}(\mathbf{K})$ & 247 & 244 & 241 & 234 & 218 \\
\hline & $\mathbf{R}^{2}$ & 0.998 & 0.980 & 0.985 & 0.994 & 0.79 \\
\hline \multirow{3}{*}{$\begin{array}{l}{ }^{* * * *} \text { Derived } \\
\text { calculations }\end{array}$} & $\mathbf{B}(\mathbf{K})$ & 1901 & 2014 & 2119 & 1915 & 2220 \\
\hline & $\mathbf{m}$ & 98,1 & 103,1 & 107,5 & 93,5 & 103,6 \\
\hline & $\begin{array}{c}\text { Ea } \\
\left(\mathbf{k J} \cdot \mathbf{m o l}^{-1}\right)\end{array}$ & 235,6 & 236,4 & 229,3 & 179,9 & 155,6 \\
\hline
\end{tabular}

* Glass transition calculated as the temperature at which the dielectric relaxation time is $100 \mathrm{~s}$.

** Calculated as (Tg_DGEBA/PETMP-Tg_thiol-epoxy/thiol-ene) / (Tg_DGEBA/PETMP- Tg_HBPEI/PETMP) X 100

*** Taken as a reference for the VFTH to obtain $\mathrm{D}$ and $\operatorname{lnf}_{0}$, as $\mathrm{Tg}-50 \mathrm{~K}$ and rounded to the closest entire number

$* * * *$ Values within a 2-5\% error margin

Concerning the mixtures of dual-cured thiol-epoxy/thiol-ene thermosets, the higher the substitution of DGEBA/T units by HBPEI/T units in the thermosets, the lower the Ea of the $\alpha$-relaxation was. All these results can be ascribed to the synergic effect of reducing the rigidity and compactness of the thermosets by the reduction of aromatic moieties of the thiol-epoxy units, and the increase of the volume per unit of functionality induced by the addition of thiol-ene units, as well as the inherent flexibility of the hyperbranched structure of HBPEI. This is in agreement with previous results obtained by DynamicMechanical-Thermal-Analysis [4], and with the modification of the glass transition temperature of the thermosets shown in Table 2. As expected, lower activation energies required lower temperatures for the dipole transfer to overcome the glass-rubber transition. There was not, however, a linear relationship between the presence of thiolene units and the reduction of $T g$. In fact, a 25\% of HBPEI/PETMP units reduced the $T g$ an $8 \%$, a $50 \%$ of HBPEI/PETMP units a $22 \%$ and a $75 \%$ of HBPEI/PETMP units a $44 \%$. Instead, this evolution could be modelled by means of a Boltzman-like sigmoidal function shown in $\boldsymbol{E} \boldsymbol{q}$ 9, where $T g_{\overline{E a}}$ represents the glass transition temperature at the average apparent activation energy of the two limiting values, i.e. the inflection point, and $S T_{T g}$ is 
a measure of the steepness of the curve. The high confidence fitting values above 0.999 of correlation coefficient, shown in Figure 9, might represent a starting point for the tailoring of compositions of thermosets, by balancing appropriate amounts of rigid and flexible moieties to obtain the desired relationship between activation energies and glass transition temperatures.

$$
E a_{\alpha}(T g)=E a_{\alpha \_H B P E I / P E T M P}+\frac{E a_{\alpha_{H B P E I / P E T M P}-E a_{\alpha_{D G E B A / P E T M P}}}}{1+e^{\left(\frac{T g-T g_{\overline{E a}}}{S T T g}\right)}}(\boldsymbol{E q} \text {. 9) }
$$

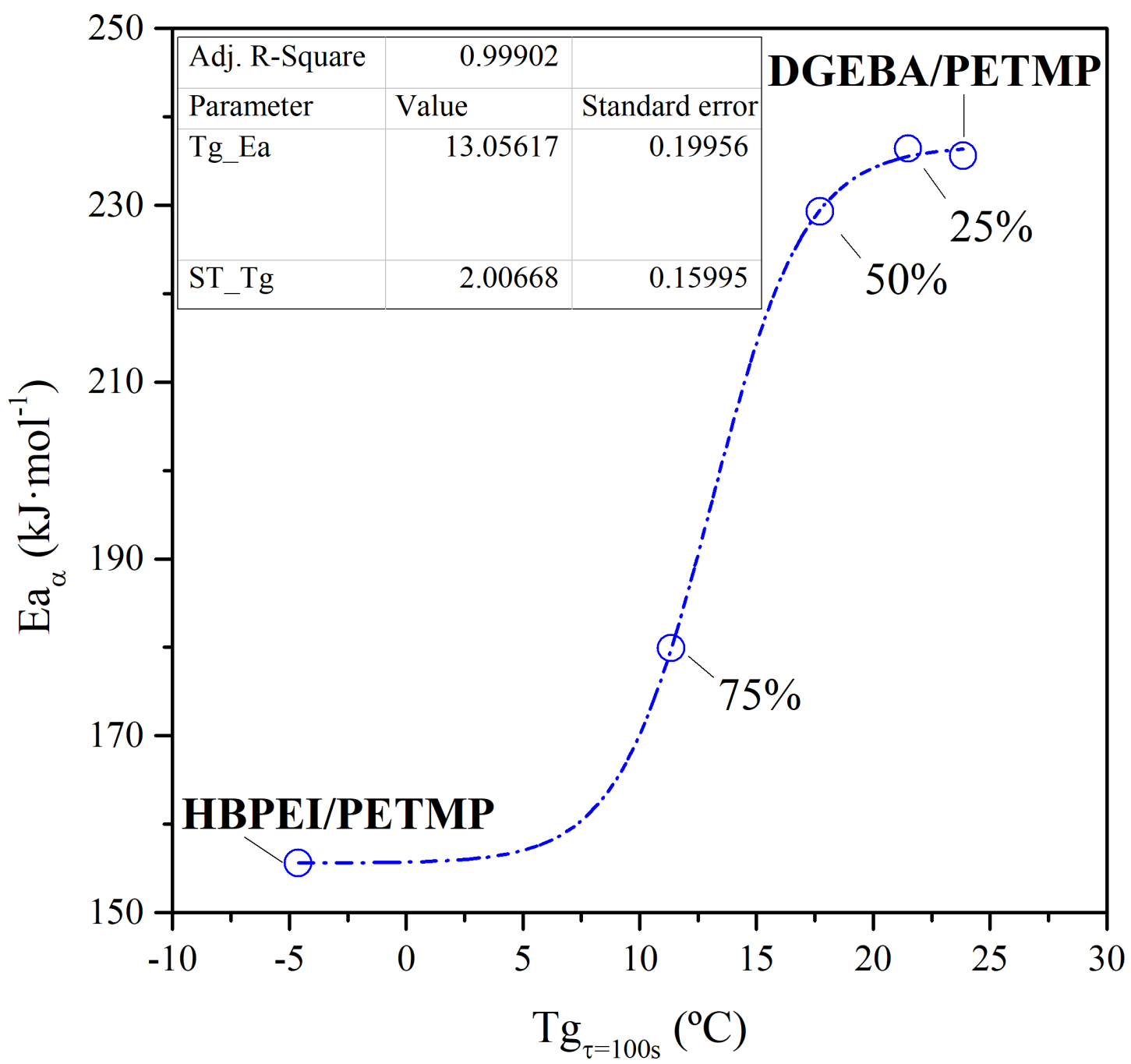

Figure 9. Boltzman-like fitting of evolution of apparent activation energy of the $\alpha$-relaxation of the thiol-epoxy/thiol-ene thermosets. 


\section{Conclusions}

The thermosets studied by dielectric spectroscopy have been obtained by a dual curing procedure consisting in a first photochemical thiol-ene reaction between the allyl terminated poly(ethyleneimine) (HBPEI) and the tetrathiol (PETMP), followed by a thermal thiol-epoxy reaction between diglycidylether of bisphenol A (DGEBA) and PETMP. The two crosslinked structures formed are linked by the PETMP, forming a unique copolymeric network, with different proportions of DGEBA/HBPEI in the formulations of the thermosets.

The dielectric spectra revealed two sub- $T g$ intramolecular non-cooperative $\gamma$ and $\beta$ relaxations and an intermolecular cooperative $\alpha$-relaxation at the $T g$.

The $\gamma$-relaxation was an intramolecular non-cooperative relaxation, ascribed to the dipole orientation of the terminal SH groups. This relaxation splitted into two sub-relaxations labelled as $\gamma_{\text {epo }}$ or $\gamma_{e n e}$, according to the predominant phase of thiol-epoxy or thiol-ene present in the thermoset. Their evolution depended on the vicinities of the functional group. The $\gamma_{\text {epo }}$ relaxation appeared for all compositions. Thermosets with a content of flexible HBPEI/PETMP domains higher than a $50 \% \mathrm{w} / \mathrm{w}$ showed the $\gamma_{\text {ene }}$ relaxation at lower temperatures and higher frequencies. There was no linear composition rule of the activation energies.

The $\beta$-relaxation was ascribed to the dipole transfer of the linear $-\mathrm{CH}_{2}-\mathrm{O}-(\mathrm{C}=\mathrm{O})-\mathrm{CH}_{2}-$ $\mathrm{CH}_{2}$ - groups of the thiol structure constrained in the glassy state. It was not a JohariGoldstein relaxation, and was not therefore connected to the subsequent $\alpha$-relaxation. It was thus ascribed to an intramolecular non-cooperative relaxation.

The $\alpha$-relaxation was an intermolecular cooperative relaxation, ascribed to the long-term segmental movements of the thermoset occurring during the transition from the glassy to the rubber stage. A synergic effect of reducing the rigidity and compactness of the thermosets by the reduction of aromatic moieties of the thiol-epoxy units, and the increase of the flexibility induced by the addition of thiol-ene units, due to both the structure of HBPEI and the increase of the volume per unit of functionality, was shown. The higher the presence of thiol-ene domains, the lower the apparent activation energy of the 
thermosets was, in agreement with the lower glass transition temperature $\mathrm{Tg}$. The evolution of the apparent activation energy of the thiol-epo / thiol-ene thermosets could be modelled by means of a sigmoidal Boltzman-like function. 


\section{Acknowledgements}

European Regional Development Funds and the Spanish Ministry of Science, Innovation and Universities for ENE2017-86711-C3-1-R, MAT2017-82849-C2-1-R, ENE201453734-C2-1-R, UPOV13-3E-1947 and projects. Ministry of Education for the FPU13/01916) for O. Gil-Castell. Generalitat de Catalunya for the 2017-SGR-77 project. Generalitat Valenciana for the APOSTD14/041 for JD Badia and GRISOLIA/2012/003 for R. Teruel-Juanes. This paper is warmly dedicated to Martina Badia-Ombuena, in commemoration of her birth.

\section{Data availability}

The raw/processed data required to reproduce these findings cannot be shared at this time as the data also forms part of an ongoing study. 


\section{References}

[1] X. Ramis, X. Fernández-Francos, S. De la Flor, F. Ferrando, and À. Serra, "Click-based dual-curing thermosets and their applications," Thermosets, pp. 511-541, Jan. 2018.

[2] A. B. Lowe, “Thiol-ene 'click' reactions and recent applications in polymer and materials synthesis," Polym. Chem., vol. 1, no. 1, pp. 17-36, 2010.

[3] D. Guzmán, X. Ramis, X. Fernández-Francos, and A. Serra, "Preparation of click thiol-ene/thiol-epoxy thermosets by controlled photo/thermal dual curing sequence," RSC Adv., vol. 5, no. 123, pp. 101623-101633, 2015.

[4] C. Acebo, X. Fernández-Francos, X. Ramis, and À. Serra, "Multifunctional allylterminated hyperbranched poly (ethyleneimine) as component of new thiolene/thiol-epoxy materials," React. Funct. Polym., vol. 99, pp. 17-25, 2016.

[5] M. Adeli and R. Haag, "Multiarm star nanocarriers containing a poly(ethylene imine) core and polylactide arms," J. Polym. Sci. Part A Polym. Chem., vol. 44, no. 19 , pp. 5740-5749, Oct. 2006.

[6] C. Acebo, A. Picardi, X. Fernández-Francos, S. De la Flor, X. Ramis, and À. Serra, "Effect of hydroxyl ended and end-capped multiarm star polymers on the curing process and mechanical characteristics of epoxy/anhydride thermosets," Prog. Org. Coatings, vol. 77, no. 8, pp. 1288-1298, 2014.

[7] E. Riande and R. Díaz-Calleja, Electrical properties of polymers. CRC Press, 2004.

[8] R. Casalini, D. Fioretto, A. Livi, M. Lucchesi, and P. A. Rolla, "Influence of the glass transition on the secondary relaxation of an epoxy resin," Phys. Rev. B, vol. 56, no. 6, p. 3016, 1997.

[9] S. Capaccioli, S. Corezzi, G. Gallone, P. A. Rolla, L. Comez, and D. Fioretto, "Dynamics of epoxies: a full dielectric analysis by wideband spectroscopy," $J$. Non. Cryst. Solids, vol. 235, pp. 576-579, 1998.

[10] S. Corezzi et al., "Two crossover regions in the dynamics of glass forming epoxy resins," J. Chem. Phys., vol. 117, no. 5, pp. 2435-2448, 2002.

[11] M. Beiner and K. L. Ngai, "Interrelation between primary and secondary relaxations in polymerizing systems based on epoxy resins," Macromolecules, vol. 38, no. 16, pp. 7033-7042, 2005.

[12] G. Gallone, S. Capaccioli, G. Levita, P. A. Rolla, and S. Corezzi, "Dielectric 
analysis of the linear polymerization of an epoxy resin," Polym. Int., vol. 50, no. 5, pp. 545-551, 2001.

[13] H. Kim and K. Char, "Dielectric changes during the curing of epoxy resin based on the diglycidyl ether of bisphenol A (DGEBA) with diamine," 1999.

[14] M. Tarnacka, M. Wikarek, S. Pawlus, K. Kaminski, and M. Paluch, "Impact of high pressure on the progress of polymerization of DGEBA cured with different amine hardeners: dielectric and DSC studies," RSC Adv., vol. 5, no. 128, pp. 105934-105942, 2015.

[15] F. Román, P. Colomer, Y. Calventus, and J. M. Hutchinson, "Molecular mobility in hyperbranched polymers and their interaction with an epoxy matrix," Materials (Basel)., vol. 9, no. 3, p. 192, 2016.

[16] L. Gao and J. Wang, "Thermal, mechanical, and dielectric studies on the DGEBA epoxy blends by using liquid oxidized poly (1, 2-butadiene) as a reactive component,” J. Appl. Polym. Sci., vol. 134, no. 15, 2017.

[17] W. Jilani et al., "A comparative study of structural and dielectric properties of diglycidyl ether of bisphenol A (DGEBA) cured with aromatic or aliphatic hardeners," J. Mater. Sci., vol. 51, no. 17, pp. 7874-7886, 2016.

[18] F. Román, P. Colomer, Y. Calventus, and J. M. Hutchinson, "Study of the molecular dynamics of multiarm star polymers with a poly (ethyleneimine) core and poly (lactide) multiarms," Materials (Basel)., vol. 10, no. 2, p. 127, 2017.

[19] F. Román, P. Colomer, Y. Calventus, and J. M. Hutchinson, "Study of Hyperbranched Poly (ethyleneimine) Polymers of Different Molecular Weight and Their Interaction with Epoxy Resin," Materials (Basel)., vol. 11, no. 3, p. $410,2018$.

[20] R. Teruel-Juanes, "Propiedades dieléctricas y conductividad de nuevos electrolitos poliméricos para aplicaciones energéticas." Universitat Politècnica de València, p. Doctoral Thesis, 2017.

[21] J. C. Maxwell, "Electricity and Magnetism, vol. 1, Clarendon." Oxford, 1892.

[22] K. W. Wagner, "The after effect in dielectrics," Arch. Electrotech, vol. 2, no. 378, p. e394, 1914.

[23] R. W. Sillars, "The properties of a dielectric containing semiconducting particles of various shapes," Inst. Electr. Eng. Wirel. Sect. Inst., vol. 12, no. 35, pp. 139$155,1937$.

[24] Z. Lu, E. Manias, and D. D. Macdonald, "Dielectric Relaxation Spectroscopy 
Studies on Water-Saturated Nafion 117 Membrane," in In Proceedings of the Electrochemical Society 204th National Meeting, 2003.

[25] G. W. Gross and J. Johnson, "The Layered Capacitor Method for Dielectric Bridge Measurements. Data Analysis and Interpretation of Fluoride Doped ICE.," IEEE Trans. Electr. Insul., no. 5, pp. 485-497, 1983.

[26] A. R. Von Hippel, "Dielectrics and waves," 1954.

[27] J. D. Badia et al., "Effect of sisal and hydrothermal ageing on the dielectric behaviour of polylactide/sisal biocomposites," Compos. Sci. Technol., vol. 149, pp. 1-10, 2017.

[28] A. M. M. Salaberría et al., "Influence of chitin nanocrystals on the dielectric behaviour and conductivity of chitosan-based bionanocomposites," Compos. Sci. Technol., vol. 167, pp. 323-330, Oct. 2018.

[29] A. Martínez-Felipe, L. Santonja-Blasco, J. D. Badia, C. T. Imrie, and A. RibesGreus, "Characterization of functionalized side-chain liquid crystal methacrylates containing nonmesogenic units by dielectric spectroscopy," Ind. Eng. Chem. Res., 2013.

[30] J. D. Badia et al., "Dielectric spectroscopy of reprocessed polylactide," Polym. Degrad. Stab., vol. 107, pp. 21-27, Sep. 2014.

[31] J. M. Charlesworth, "Deconvolution of overlapping relaxations in dynamic mechanical spectra," J. Mater. Sci., vol. 28, no. 2, pp. 399-404, 1993.

[32] S. Havriliak and S. Negami, "A complex plane representation of dielectric and mechanical relaxation processes in some polymers," Polymer (Guildf)., vol. 8, pp. 161-210, 1967.

[33] S. Havriliak and S. Negami, "A complex plane analysis of $\alpha$-dispersions in some polymer systems," in Journal of Polymer Science Part C: Polymer Symposia, 1966, vol. 14, no. 1, pp. 99-117.

[34] H. Eyring, "The activated complex in chemical reactions," J. Chem. Phys., vol. 3, no. 2, pp. 107-115, 1935.

[35] J. Heijboer, "Molecular origin of relaxations in polymers," Ann. N. Y. Acad. Sci., vol. 279, no. 1, pp. 104-116, 1976.

[36] J. Heijboer, "Secondary loss peaks in glassy amorphous polymers," in Molecular basis of transitions and relaxations, D. J. Meier, Ed. CRC Press, 1978, p. 429.

[37] H. Vogel, "The temperature dependence law of the viscosity of fluids," Phys. Z, vol. 22, pp. 645-646, 1921. 
[38] G. S. Fulcher, "Analysis of recent measurements of the viscosity of glasses," $J$. Am. Ceram. Soc., vol. 75, no. 5, pp. 1043-1055, 1992.

[39] C. A. Angell, "Formation of glasses from liquids and biopolymers," Science (80-. )., vol. 267, no. 5206, pp. 1924-1935, 1995.

[40] S. A. Cruz and M. Zanin, "Dielectric strength of the blends of virgin and recycled HDPE,” J. Appl. Polym. Sci., vol. 91, no. 3, pp. 1730-1735, 2004.

[41] W. Zheng and S.-C. Wong, "Electrical conductivity and dielectric properties of PMMA/expanded graphite composites," Compos. Sci. Technol., vol. 63, no. 2, pp. 225-235, 2003.

[42] S. Singha and M. J. Thomas, "Dielectric properties of epoxy nanocomposites," IEEE Trans. Dielectr. Electr. Insul., vol. 15, no. 1, pp. 12-23, 2008.

[43] A. Garcia-Bernabé, R. Díaz-Calleja, and R. Haag, "Broadband dielectric spectroscopy studies of hyperbranched polyglycerols," Macromol. Chem. Phys., vol. 207, no. 11, pp. 970-977, 2006.

[44] A. Garcia-Bernabé, G. Dominguez-Espinosa, R. Diaz-Calleja, E. Riande, and R. Haag, "Secondary and primary relaxations in hyperbranched polyglycerol: A comparative study in the frequency and time domains," J. Chem. Phys., vol. 127, no. 12, p. 124904, 2007.

[45] A. Kyritsis, K. Raftopoulos, M. A. Rehim, S. S. Shabaan, A. Ghoneim, and G. Turky, "Structure and molecular dynamics of hyperbranched polymeric systems with urethane and urea linkages," Polymer (Guildf)., vol. 50, no. 16, pp. 40394047, 2009.

[46] C. Hakme et al., "Dielectric studies of hyperbranched aromatic polyamide and polyamide-6, 6 blends," J. Appl. Polym. Sci., vol. 97, no. 4, pp. 1522-1537, 2005.

[47] G. Turky, J. R. Sangoro, M. Abdel Rehim, and F. Kremer, "Secondary relaxations and electrical conductivity in hyperbranched polyester amides," $J$. Polym. Sci. Part B Polym. Phys., vol. 48, no. 14, pp. 1651-1657, 2010.

[48] G. P. Johari and M. Goldstein, "Viscous Liquids and the Glass Transition. II. Secondary Relaxations in Glasses of Rigid Molecules," J. Chem. Phys., vol. 53, no. 6 , pp. $2372-2388,1970$.

[49] K. L. Ngai and M. Paluch, "Classification of secondary relaxation in glassformers based on dynamic properties," J. Chem. Phys., vol. 120, no. 2, pp. 857873, 2004.

[50] E. Malmström, A. Hult, U. W. Gedde, F. Liu, and R. H. Boyd, "Relaxation 
processes in hyperbranched polyesters: Influence of terminal groups," Polymer (Guildf)., vol. 38, no. 19, pp. 4873-4879, 1997.

[51] K. Kunal, C. G. Robertson, S. Pawlus, S. F. Hahn, and A. P. Sokolov, "Role of chemical structure in fragility of polymers: A qualitative picture," Macromolecules, vol. 41, no. 19, pp. 7232-7238, 2008.

[52] Q. Qin and G. B. McKenna, "Correlation between dynamic fragility and glass transition temperature for different classes of glass forming liquids," J. Non. Cryst. Solids, vol. 352, no. 28-29, pp. 2977-2985, Aug. 2006.

[53] J. R. Sangoro et al., "Charge transport and dipolar relaxations in hyperbranched polyamide amines," Macromolecules, vol. 42, no. 5, pp. 1648-1651, 2009. 